\title{
Autoimmune central diabetes insipidus in a patient with ureaplasma urealyticum infection and review on new triggers of immune response
}

${ }^{1}$ Clinical Immunology, Department of Internal Medicine and Medical Specialties (DiMI), University of Genoa, Italy ${ }^{2}$ Nephrology, Department of Interna Medicine and Medical Specialties (DiMI), University of Genoa, Italy

${ }^{3}$ Endocrinology Units, Department of Internal Medicine and Medical Specialties

(DiMI), University of Genoa, Italy

${ }^{4}$ Center of Excellence for

Biomedical Research (CEBR)

${ }^{5}$ Clinical Neurology Unit, Department of Neurosciences, Rehabilitation,

Ophthalmology, Genetics and Maternal and Child Health, IRCCS AOU San

Martino-IST, University of Genoa, Italy ${ }^{6}$ Endocrinology Unit, Department of

Clinical and Experimental Medicine and

Surgery "F. Magrassi-A. Lanzara", Second

University of Naples, Naples, Italy

Correspondence to:

Giuseppe Murdaca

Department of Internal Medicine

and Medical Specialties (DiMI)

Clinical Immunology Unit

Viale Benedetto XV, 6

16132 - Genova, Italy

Giuseppe.murdaca@unige.it

Received on May/18/2015

Accepted on May/27/2015

DOI: 10.1590/2359-3997000000072

\author{
Giuseppe Murdaca', Rodolfo Russo², Francesca Spanò', \\ Diego Ferone ${ }^{3,4}$, Manuela Albertelli ${ }^{3,4}$, Angelo Schenone ${ }^{5}$, \\ Miriam Contatore', Andrea Guastalla', Annamaria De Bellis', \\ Giacomo Garibotto², Francesco Puppo'
}

\begin{abstract}
SUMMARY
Diabetes insipidus is a disease in which large volumes of dilute urine (polyuria) are excreted due to vasopressin (AVP) deficiency [central diabetes insipidus (CDI)] or to AVP resistance (nephrogenic diabetes insipidus). In the majority of patients, the occurrence of $\mathrm{CDI}$ is related to the destruction or degeneration of neurons of the hypothalamic supraoptic and paraventricular nuclei. The most common and well recognized causes include local inflammatory or autoimmune diseases, vascular disorders, Langerhans cell histiocytosis (LCH), sarcoidosis, tumors such as germinoma/craniopharyngioma or metastases, traumatic brain injuries, intracranial surgery, and midline cerebral and cranial malformations. Here we have the opportunity to describe an unusual case of female patient who developed autoimmune CDI following ureaplasma urealyticum infection and to review the literature on this uncommon feature. Moreover, we also discussed the potential mechanisms by which ureaplasma urealyticum might favor the development of autoimmune CDI. Arch Endocrinol Metab. 2015;59(6):554-8
\end{abstract}

\section{INTRODUCTION}

D iabetes insipidus (DI) is a disabling and rather often severe disease in which large volumes of dilute urine (polyuria) are excreted due to posterior pituitary and antidiuretic hormone vasopressin (AVP) deficiency [central diabetes insipidus (CDI)], or to peripheral AVP resistance (nephrogenic diabetes insipidus). DI is accompanied by reactive polydipsia and very high risk of dehydration (1). The destruction or degeneration of neurons, originating in the hypothalamic supraoptic and paraventricular nuclei, produce the disease in the majority of patients. The main and well recognized causes of CDI (Table 1) include local inflammatory or autoimmune diseases, vascular disorders, Langerhans cell histiocytosis (LCH), sarcoidosis, germinoma/craniopharyngioma, metastases, trauma resulting from surgery or accidents, and midline cerebral, as well as cranial malformations (2). In rare cases, the underlying cause can be a genetic defect in AVP synthesis that could be inherited as autosomal dominant, autosomal recessive or X-linked recessive traits (1). Although CDI has been reported to be idiopathic in $30-50 \%$ of cases, the identification of antibodies against AVP-secreting neurons $(3,4)$, as well as the use of more modern imaging techniques, have made the true idiopathic form an uncommon finding (2). Indeed, more recently, it has been noted that some patients in the acute phase develop lymphocytic infundibuloneurohypophysitis (LINH), suggesting that an autoimmune mechanism is involved in the development of the disease (5).

Here we first describe an unusual case of female patient, who developed autoimmune CDI and reactive arthritis, following ureaplasma urealyticum (UU) infection, and we took this opportunity to review the current literature concerning this type of DI. We finally discussed the potential mechanisms by which UU might have favored the development of CDI in this specific case. 
Table 1. Causes of diabetes insipidus

\begin{tabular}{ll}
\hline Central & Nephrogenic \\
\hline Surgery & Drugs \\
Transcranial NCH & Lithium \\
Transsphenoidal NCH & Ofloxacin \\
Cranial trauma/Brain injury & Demeclocycline \\
Primary tumors & Amphotericin B \\
Hypothalamic tumor & Aminoglycosides \\
Craniopharyngioma & Cisplatin \\
Meningioma & Cidofovir \\
Dysgerminoma & Foscarnet \\
Glioma & Didanosine \\
Haematologic disorders & Ifosfamide \\
Lymphoma & Obstructive \\
Leukemia & Sarcoma \\
Pituitary metastases & Vascular \\
Breast & Sickle cell disease and trait \\
Lung & Acute tubular necrosis \\
Prostate & Metabolic \\
NET & Severe hypercalcemia \\
Infections & Severe hypokalemia \\
Tuberculosis (meningitis) & Infiltrative diseases \\
Viral infections (meningitis) & Amyloidosis \\
Intracranial abscess & Sjögren's syndrome \\
Toxoplasmosis & Granulomatous diseases \\
Granulomatous diseases & Sarcoidosis \\
Tuberculosis (mass effect) & Genetic \\
Sarcoidosis & \\
Histiocytosis & \\
Inflammatory & \\
Systemic lupus erythematosus & \\
Scleroderma & \\
Wegener's disease & \\
Vascular & \\
Aneurysm & \\
Hypoxic encephalopathy & \\
Sheehan's syndrome & \\
Chemicals & \\
Snake venom & \\
Tetrodotoxin & \\
\hline
\end{tabular}

NCH: neurosurgery; NET: neuroendocrine tumors.

\section{CASE REPORT}

On January 2011, a 45 year-old-female was admitted for arthralgia, swelling and limited movement of hands, feet and knees lasting for 6 months. The patient was experiencing polydipsia, polyuria and nicturia since November 2010. Indeed, she was drinking about 7-8 L of water per day, and urinated every 1-2 hours at night with a daily urinary output of approximately $7 \mathrm{~L}$. Urine specimens were cultured for aerobic bacterial species and examined by polymerase chain reaction-based assays for the presence of chlamydia trachomatis, mycoplasma hominis, ureaplasma parvum and UU. Among these latter, only UU was detected. Elevated serum antibody titer to UU was demonstrated and UU was isolated from the knee synovial fluid as well. Antinuclear antibodies (ANA), anti-neutrophil cytoplasmic antibodies (ANCA), anti-cardiolipin, anti- $\beta_{2}$-glycoprotein-I and anti-phosphatidylserine antibodies, lupus anticoagulant (LAC), human leucocytes antigen (HLA)-B51, HLA-B27, rheumatoid factor test and anticitrulline antibodies, anti-thyroid peroxidase antibody, anti-thyroglobulin antibodies and thyroid-stimulating hormone receptor antibody were negative. Fasting blood glucose was $75 \mathrm{mg} / \mathrm{dL}$, glycated hemoglobin (HbAlc) was $4.9 \%$ (reference value: $4.3-5.8 \%$ ), urinalysis was unremarkable and did not show any evidence of glycosuria, which excluded the possibility of symptomatic diabetes mellitus. A further endocrinological assessment showed low level of antidiuretic hormone (AVP; $0.7 \mathrm{pg} / \mathrm{mL}$ ) and normal levels of anterior pituitary hormones, as well as cortisol, free T3 and T4. The patient underwent the water deprivation test and plasma osmolality was 380 $\operatorname{mosm} / \mathrm{Kg}$, at baseline, while urine osmolality was 152 mosm $/ \mathrm{Kg}$. After 7 hours of water deprivation, plasma osmolality was $388 \mathrm{mosm} / \mathrm{Kg}$ and urine osmolality was $140 \mathrm{mosm} / \mathrm{Kg}$. After the administration of five units of 1-deamino-8-D-arginine vasopressin (DDAVP), urine osmolality raised to $780 \mathrm{mosm} / \mathrm{Kg}$, strongly indicating the presence of CDI. The diagnosis was confirmed by magnetic resonance (MR) imaging, which displayed the absence of classical posterior pituitary lobe hyperintensity in Tl. Anti-pituitary antibodies (APAs) resulted negative while anti-hypothalamus antibodies (AHAs) were positive. AHAs and APAs were detected by simple indirect immunofluorescence method on cryostat sections of young baboon hypothalamus and pituitary, respectively, supplied by Biosystem Italia Srl (San Martino Buon Albergo, VR, Italy), as described previously (6). Finally, quantiferon tuberculosis gold test resulted negative and angiotensin-converting enzyme (ACE) was in the normal range. The diagnosis of an autoimmune CDI and reactive arthritis following UU infection was made and the patient started oral desmopressin $(100 \mathrm{mcg}$ at bedtime), experiencing a marked relief of polyuria, as well as polydipsia. Finally, the reactive arthritis was treated with doxycycline (100 mg twice daily for 14 days), followed by a single $2 \mathrm{~g}$ dose of azithromycin. Complete clinical remission of arthritis was achieved after ten days of tre- 
atment. The patients signed an informed consent for the use of the data concerning the case.

\section{DISCUSSION AND UPDATE UPON CURRENT LITERATURE}

The identification of antibodies against AVP-secreting cells (AVPc) on the one hand (3), and the advances in imaging techniques on the other (4), have shed light on the pathophysiological aspects of CDI, making the idiopathic form a very uncommon condition. An autoimmune process involving the hypothalamic-neuroendocrine AVPc leading to CDI was initially suggested in the early 1980s by Scherbaum and Bottazzo (7). Subsequently, AVPc autoantibodies were detected in $37 \%$ of individuals affected by idiopathic CDI and in $6.3 \%$ of those with CDI associated with $\mathrm{LCH}$, at a mean age of 34.9 years (4). Furthermore, Pivonello and cols. reported a relationship between AVPc autoantibodies and clinical, immunological and radiological features in about $23 \%$ of a large cohort of individuals with CDI of different etiologies at mean age of 29.2 years (8). All these findings confirm that autoimmune CDI most commonly affects female patients with associated autoimmune diseases. The evidence of pituitary stalk thickening, as well as the absence of classical posterior pituitary lobe hyperintensity in $\mathrm{Tl}$ at $\mathrm{MR}$ imaging, generally characterizes the radiologic features of these patients. Finally, AVPc autoantibodies have been reported in patients affected by autoimmune polyendocrinopathy (9), while pituitary stalk thickening and CDI have been described in the course of autoimmune polyglandular syndrome (2). The fact that AVPc autoantibodies are recognized in few patients seems to indicate either that they are subject to early disappearance or, possibly, that autoimmune T-cell local damage took place, not, however, necessarily associated with autoantibody formation (4). Indeed, Imura and cols. confirmed the immune-mediated pathogenesis of some cases of CDI using neuroimaging and histological assessment (5). These authors reported that an autoimmune process, affecting the posterior pituitary gland and infundibulum, without anterior pituitary involvement, could cause LINH. Furthermore, hypothalamic-neurohypophyseal autoimmune involvement seems to occur more commonly in children and young adults with idiopathic CDI (4). However, a high frequency of AVPc autoantibodies was observed in patients with LCH characterized by "activated antigen presenting cells" (in particular histiocytes) in the target-affec- ted tissue (10). Patients with LCH and CDI presented radiological and immunological targets similar to those with idiopathic CDI, suggesting that both diseases may have a common pathogenesis. The hypothesis of an important role of autoimmunity in the pathogenesis of CDI is strengthened by the fact that the pituitary gland is susceptible to CD8+ T-cell-mediated autoimmunity, triggered by a cell-specific model autoantigen (11), as well as to the development of autoimmune hypophysitis through the immunization of female SJL/J mice with mouse pituitary extracts (12). Furthermore, LINH has been found associated also with autoimmune inflammatory disease of the pituitary gland, although the exact etiology remains unknown so far. The underlying process of pituitary stalk thickening in idiopathic CDI is not yet completely understood. The term LINH has been introduced to distinguish between children and adolescents with CDI (2), pituitary hormone deficiency, decrease in anterior pituitary size and transient and/or persistent pituitary stalk thickening and adult subjects with similar posterior pituitary findings at MR imaging, but normal size and function of the anterior pituitary (5). Mirocha and cols. reported two different potential pathogenic mechanisms (13). The first one apparently directed against self-antigens and known as "T-helper dominance", whereas the second one directed against non-self-antigens (i.e., post-infection mechanism). Both these mechanisms may favor primary hypophysitits.

However, LINH is considered a disease characterized by lymphocytic infiltration of the posterior lobe of the pituitary gland, resulting in clinically evident CDI. It has been suggested that an immune response, triggered by viral or bacterial infection, could be involved in the development of LINH. However, the viruses or bacteria that may commonly cause this disease have not yet been identified, probably because of the extremely low incidence. Kobayashi and cols. reported a case of CDI following probable type A/ HINl flu infection (14). In this case, the autoimmune mechanism of LINH was hypothesized associated with novel flu sustained by A/HINl virus infection (14). Furthermore, cases of LINH after meningoencephalitis due to the type A flu virus, or herpes simplex virus, were previously reported (15). Hannon and cols. a retrospectively reviewed the databases from the endocrinology units of two tertiary referral centers, in England and Ireland, and identified 39 patients with CDI, who presented other autoimmune diseases, such as Hashimoto's thyroiditis, Graves' disease, type 1 
diabetes mellitus and Addison's disease, including vitamin B12 deficiency secondary to Addisonian pernicious anaemia (16). These data were in line with an estimated overall incidence of $28-38 \%$ for associated autoimmune endocrine disease in autoimmune CDI, as defined by the presence of AVPc autoantibodies (810). Finally, in the present case, there was a temporal association between the UU infection and the onset of polyuria and polydipsia. To our knowledge, this is the first case of autoimmune CDI following UU infection. The pathogenic link remains obscure although an immunologic response to UU antigen may be associated with the generation of AVPc-specific antibodies, suggesting the presence of common antigenic epitopes in their structures, as reported for chlamydia trachomatis and human apolipoprotein B (17). In support of this hypothesis, infection with a pathogen sharing similar structures with autoantigens is one possibility of how pathogens might induce or accelerate autoimmunity. Such 'molecular mimicry', indeed, exists and has been detected between pathogens and autoantigens recognized by antibodies or $\mathrm{T}$ cells of patients with a broad variety of autoimmune diseases $(18,19)$. Notably, an association between the infection with campylobacter jejuni and the Guillain Barré syndrome has been described as well (20). Indeed, campylobacter jejuni shares a structural homology of the lipo- oligosaccharide with the peripheral nerve GMl ganglioside, and could be convincingly reproduced in an animal model (20). However, the best example of post-infectious autoimmunity due to molecular mimicry has been established for streptococcus pyogenes-induced acute rheumatic fever, where the lysoganglioside of the host shares a structural similarity to $\mathrm{N}$-acetyl-b-D-glucosamine, the dominant epitope of the group A streptococcal carbohydrate (21). The occurrence of "molecular mimicry" between trigger and target seems to circumvent peripheral tolerance to the target antigen, resulting in the generation of a high frequency of target antigen-specific $\mathrm{T}$ cells, which surpasses a critical threshold for the induction, or the acceleration, of autoimmune diseases. However, besides genetic predisposition and environmental factors, local inflammation seems to play a key role in the transition from autoimmunity to autoimmune overt disease. Indeed, the balance between pro- and anti-inflammatory chemokines and cytokines, which determines the local inflammatory milieu, or the proportion of apoptotic and anti-apoptotic signals influencing the fate of a cell, the ratio between aggressive and regulatory T-lymphocytes might be as important as the absolute number of autoaggressive lymphocytes (22). The case we presently reported represents an example of pathogenetic autoimmune pathway induced by a cross-reactive immune response following UU, and confirms that autoimmune CDI may be included among the potential clinical manifestations of cross-reactive response in individuals exposed to UU infection.

\section{TREATMENT OF CENTRAL DIABETES INSIPIDUS}

The drug of choice for the treatment of CDI is DDAVP (desmopressin). Given intranasally or currently more often orally, maximum plasma concentration is reached in about 40-55 minutes and its half-life is about 3-5 hours. The intravenous route is only used in case of disabled patients, coma, or other condition contraindicating or impeding the other modalities of treatment. Orally daily dosages may vary from 100 to $1200 \mu \mathrm{g}$ (once or twice a day). Symptomatic dilution hyponatremia is a potential hazard if desmopressin is administered in excess over a long period. Symptoms of hyponatremia include headache, nausea, vomiting, seizure and, in untreated cases, coma and death. Notably, extrapontine myelinosis may occur in multidrug treated individuals (23). Corticosteroid treatment of LINH gave conflicting results (14). However, starting corticosteroid therapy as soon as possible seems to improve more rapidly the clinical picture of CDI (14). It is advisable to start treatment within 2 months from symptom onset. Indeed, Kajiyama and cols. described a case of a patient with clinical autoimmune CDI associated with systemic lupus erythematosus (SLE) and dermatomyositis (24). After treatment with intravenous cyclophosphamide and concomitant oral prednisone, AVPc antibodies disappeared, CDI improved and vasopressin replacement therapy was completely withdrawn. Finally, TNF- $\alpha$ inhibitors appeared efficient in the treatment of chronic immune-mediated or inflammatory diseases, such as rheumatoid arthritis, ankylosing spondylitis, psoriasis and/or psoriatic arthritis (25-31). Which may be their potential effectiveness in treating autoimmune CDI, is a question that remains unanswered so far. However, tumor necrosis factor- $\alpha$ inhibitors may represent a new frontier in the treatment of autoimmune CDI associated with systemic autoimmune diseases.

Disclosure: no potential conflict of interest relevant to this article was reported. 


\section{REFERENCES}

1. Di lorgi N, Napoli F, Allegri AE, Olivieri I, Bertelli E, Gallizia A, et al. Diabetes insipidus - diagnosis and management. Horm Res Paediatr. 2012;77(2):69-84.

2. Maghnie M, Cosi G, Genovese E, Manca-Bitti ML, Cohen A, Zecca $S$, et al. Central diabetes insipidus in children and young adults. N Engl J Med. 2000;343(14):998-1007.

3. De Bellis A, Kelestimur F, Sinisi AA, Ruocco G, Tirelli G, Battaglia $M$, et al. Anti-hypothalamus and anti-pituitary antibodies may contribute to perpetuate the hypopituitarism in patients with Sheehan's syndrome. Eur J Endocrinol. 2008;158(2):147-52.

4. Maghnie M, Ghirardello S, De Bellis A, di lorgi N, Ambrosini L, Secco A, et al. Idiopathic central diabetes insipidus in children and young adults is commonly associated with vasopressin-cell antibodies and markers of autoimmunity. Clin Endocrinol (Oxf). 2006;65(4):470-8.

5. Imura H, Nakao K, Shimatsu A, OgawaY, SandoT, Fujisawa I, et al. Lymphocytic infundibuloneurohypophysitis as a cause of central diabetes insipidus. N Engl J Med. 1993;329(10):683-9.

6. Bellastella G, Bizzarro A, Aitella E, Barrasso M, Cozzolino D, Di Martino $S$, et al. Pregnancy may favour the development of severe autoimmune central diabetes insipidus in women with vasopressin cell antibodies: description of two cases. Eur J Endocrinol. 2015;172(3):K11-7.

7. Scherbaum WA, Bottazzo GF. Autoantibodies to vasopressin cells in idiopathic diabetes insipidus: evidence for an autoimmune variant. Lancet. 1983;321(8330):897-901.

8. Pivonello R, De Bellis A, Faggiano A, Di Salle F, Petretta M, Di Somma $C$, et al. Central diabetes insipidus and autoimmunity: relationship between the occurrence of antibodies to arginine vasopressin-secreting cells and clinical, immunological, and radiological features in a large cohort of patients with central diabetes insipidus of known and unknown etiology. J Clin Endocrinol Metab. 2003;88(4):1629-36.

9. Betterle C, Dal Pra C, Mantero F, Zanchetta R. Autoimmune adrenal insufficiency and autoimmune polyendocrine syndromes: autoantibodies, autoantigens, and their applicability in diagnosis and disease prediction. Endocr Rev. 2002;23(3):327-64.

10. Scherbaum WA, Wass JA, Besser GM, Bottazzo GF, Doniach D. Autoimmune cranial diabetes insipidus: its association with other endocrine diseases and with histiocytosis X. Clin Endocrinol (Oxf). 1986;25(4):411-20.

11. De Jersey J, Carmignac D, Le Tissier P, Barthlott T, Robinson I, Stockinger B. Factors affecting the susceptibility of the mouse pituitary gland to CD8 T-cell-mediated autoimmunity. Immunology. 2004;111(3):254-61.

12. Tzou SC, Lupi I, Landek M, Gutenberg A,Tzou YM, Kimura H, et al. Autoimmune hypophysitis of SJL mice: clinical insights from a new animal model. Endocrinology. 2008;149(7):3461-9.

13. Mirocha $S$, Elagin RB, Salamat $S$, Jaume JC. T regulatory cells distinguish two types of primary hypophysitis. Clin Exp Immunol. 2009;155(3):403-11.

14. Kobayashi T, Miwa T, Odawara M. A case of central diabetes insipidus following probable type $\mathrm{A} / \mathrm{H} 1 \mathrm{~N} 1$ influenza infection. Endocr J. 2011;58(10):913-8.
15. Kupari M, Pelkonen R, Valtonen V. Post-encephalitic hypothalamic-pituitary insufficiency. Acta Endocrinol (Copenh). 1980;94(4):433-8.

16. Hannon MJ, Orr C, Moran C, Behan LA, Agha A, Ball SG, et al. Anterior hypopituitarism is rare and autoimmune disease is common in adults with idiopathic central diabetes insipidus. Clin Endocrinol (Oxf). 2012;76(5):725-8.

17. Petyaev IM, Zigangirova NA, Tsibezov VV, Ross A, BashmakovYK. Monoclonal antibodies against lipopolysaccharide of Chlamydia trachomatis with cross reactivity to human ApoB. Hybridoma (Larchmt). 2011;30(2):131-6.

18. Srinivasappa J, Saegusa J, Prabhakar BS, Gentry MK, Buchmeier MJ, Wiktor TJ, et al. Molecular mimicry: frequency of reactivity of monoclonal antiviral antibodies with normal tissues. J Virol. 1986;57(1):397-401.

19. Christen $U$, von Herrath MG. Induction, acceleration or prevention of autoimmunity by molecular mimicry. Mol Immunol. 2004;40(14-15):1113-20.

20. Ang CW, Jacobs BC, Laman JD. The Guillain-Barré syndrome: a true case of molecular mimicry. Trends Immunol. 2004;25(2):61-6.

21. Kirvan CA, Swedo SE, Heuser JS, Cunningham MW. Mimicry and autoantibody-mediated neuronal cell signaling in Sydenham chorea. Nat Med. 2003;9(7):914-20.

22. Christen $U$, Hintermann $E$, Holdener M, von Herrath MG. Viral triggers for autoimmunity: is the 'glass of molecular mimicry' half full or half empty? J Autoimmun. 2010;34(1):38-44.

23. Maghnie M, Genovese E, Lundin S, Bonetti F, Arico M. latrogenic [corrected] extrapontine myelinolysis in central diabetes insipidus: are cyclosporine and 1-desamino-8-D-arginine vasopressin harmful in association? J Clin Endocrinol Metab. 1997;82(6):1749-51.

24. Kajiyama H, Terai C, De Bellis A, Bizzarro A, Bellastella A, Ohta S, et al. Vasopressin cell antibodies and central diabetes insipidus in a patient with systemic lupus erythematosus and dermatomyositis. J Rheumatol. 2004;31(6):1218-21.

25. Puppo F, Murdaca G, Ghio M, Indiveri F. Emerging biologic drugs for the treatment of rheumatoid arthritis. Autoimmun Rev. 2005;4(8):537-41.

26. Murdaca G, Colombo BM, Puppo F. Anti-TNF-alpha inhibitors: a new therapeutic approach for inflammatory immune-mediated diseases: an update upon efficacy and adverse events. Int J Immunopathol Pharmacol. 2009;22(3):557-65.

27. Murdaca G, Colombo BM, Barabino G, Caiti M, Cagnati P, Puppo F. Anti-tumor necrosis factor- $\alpha$ treatment with infliximab for disseminated granuloma annulare. Am J Clin Dermatol. 2010;11(6):437-9.

28. Murdaca G, Colombo BM, Puppo F. Adalimumab for the treatment of immune-mediated diseases: an update on old and recent indications. Drugs Today (Barc). 2011;47(4):277-88.

29. Murdaca G, Colombo BM, Puppo F. Emerging biological drugs: a new therapeutic approach for Systemic Lupus Erythematosus. An update upon efficacy and adverse events. Autoimmun Rev. 2011;11(1):56-60.

30. Murdaca G, Colombo BM, Cagnati P, Gulli R, Spanò F, Puppo F. Update upon efficacy and safety of TNF- $\alpha$ inhibitors. Expert Opin Drug Saf. 2012;11(1):1-5.

31. Murdaca G, Spanò F, Puppo F. Selective TNF- $\alpha$ inhibitor-induced injection site reactions. Expert Opin Drug Saf. 2013;12(2):187-93. 